\title{
Academic Delay of Gratification and its Relationship to Motivational Deter- minants, Academic Achievement, and Study Hours among Omani High School Students: A Path Analysis
}

Sabry M. Abd-El-Fattah* \& Sahar El Shourbagi

Sultan Qaboos University, Sultanate of Oman

Received: $21 / 4 / 2015$

Revised: 20/8/2015

Accepted: 20/9/2015

\begin{abstract}
This study was aimed at investigating the relationships of academic delay of gratification to motivational determinants, academic achievement, and study hours. The sample of the study included 200 Omani high school students. A path analysis showed that motivational determinants were positively related to academic delay of gratification which in turn was positively related to academic achievement and study hours. A mediational analysis showed that academic delay of gratification mediated the relationships among motivational determinants and academic achievement and study hours. There were significant gender differences in academic delay of gratification which favored females.
\end{abstract}

Keywords: Motivational determinants, academic delay of gratification, academic achievement, study hours, high school students

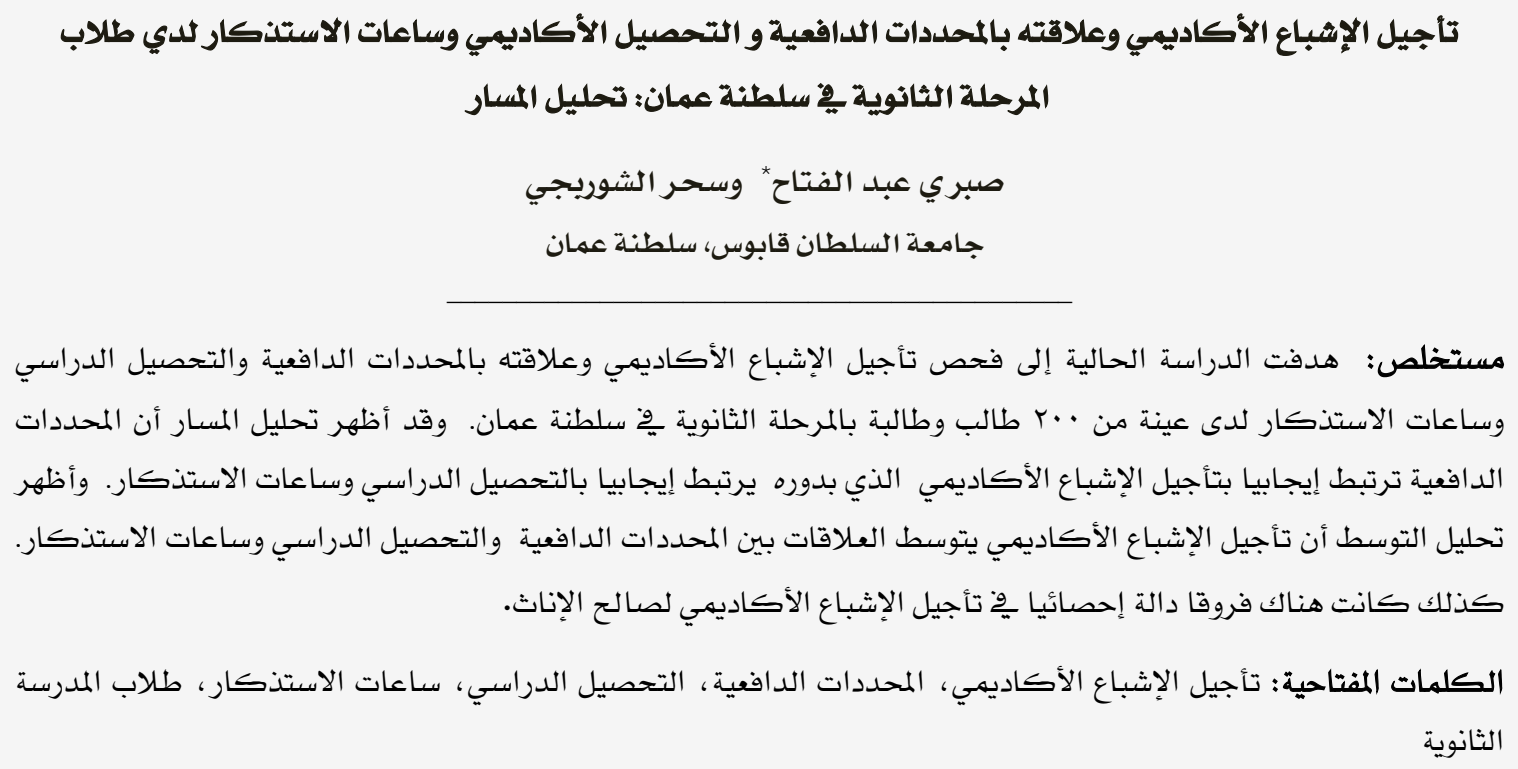




\section{A path analysis}

Beginning in the late 1960s and continuing into the early 1970s, Walter Mischel and his colleagues (Mischel, 1961; Mischel, Ebbesen, \& Zeiss, 1972) conducted a series of studies, known as the Stanford marshmallow experiment, to measure children's willpower to defer gratification. In these experiments, a preschooler would be given two marshmallows if she waited until the researcher returned to the room. The length of time individual children waited until ringing the bell was taken as a measure of their ability to delay gratification. According to Mischel and his colleagues (Mischel, 1981; Mischel \& Metzner, 1962; Mischel, Shoda, \& Peake, 1988), delay of gratification represents people's attempts to delay an attractive, immediately obtainable goal (e.g., get one marshmallow immediately) with that of pursuing long-range objectives (e.g., wait for few minutes and get two marshmallows). Delay of gratification has been conceived as an ability or competence (Mischel et al., 1988) that children develop over time and as a relatively stable generalized disposition (Funder, Block, \& Block, 1983).

From an academic perspective, many students strive to remain goal oriented and committed to tasks while facing distractions that are typical features of learning contexts. These distractions may include turning to more enjoyable activities such as going out to a party with friends, shopping, and going to the cinema or theater. Because remaining goal oriented and committed to tasks often involves foregoing an attractive, immediately obtainable goal (e.g., going to a party) in order to pursue long-range academic objectives (e.g., obtain a high score on a test), this process can be linked to a delay of gratification (Mischel 1961, 1981). Bembenutty and Karabenick (1998, p. 329) defined academic delay of gratification as students postponement of immediately available opportunities to satisfy impulses in favor of pursuing chosen important academic rewards or goals that are temporally remote but ostensibly more valuable."

\section{Gender differences in academic delay of gratification}

With respect to gender differences and their impact on academic delay of gratification, Bembenutty and Karabenick (1998) reported that female college students had higher levels of academic delay of gratification than their male counterparts. Likewise, Bembenutty (2007) found in a sample of college students that minority females had higher levels of academic delay of gratification than Caucasian males. Bembenutty (2009) reported that gender was a significant predictor of academic delay of gratification of college students with female students reporting higher levels of academic delay of gratification than their male counterparts. In addition, Villarroel (2008), using a sample of Spanish undergraduates, found that females reported higher levels of academic delay of gratification than their male counterparts. This is confusing as first Bembenutty states gender is a significant predictor then in this sentence he does not. Perhaps make it clear that this was an earlier study.

\section{Academic delay of gratification and motiva- tional determinants}

One important framework that proves to be helpful in explaining learners' preferences for diverse alternatives of action in an academic delay of gratification situation is the expectancy-value theory (Eccles, 2005, 2007; Wigfield, \& Eccles, 2000). In this theory, achievement outcomes, such as task performance and future aspirations, are primarily influenced by internalized perceptions of outcome expectancies and the value of specific tasks or domains. The expectancy component corresponds to beliefs about one's own competence and selfefficacy. The value component refers to the reasons for engaging in a specific task and includes four principal components: attainment value, intrinsic value, utility value, and cost. Attainment value is defined as the personal importance of doing well on a task, whereas intrinsic value refers to the enjoyment an individual gets from performing an activity, or to the subjective interest an individual has in a subject or activity. Utility value is determined by how well a task or domain relates to current and future goals, such as career goals and academic aspirations. Finally, cost is conceptualized in terms of the negative aspects of engaging in a task, such as performance anxiety and fear of both failure and success, as well as the amount of effort needed to succeed and the lost opportunities that result from making one choice rather than another (Eccles, 2005; Eccles \& Wigfield, 2002).

Mischel (1996) has assessed motivational determinants of delay of gratification, such as relevance, value, and expectancy for an im- 
mediate reward versus delayed reward option. His research has suggested that students' willingness to delay gratification depends upon the relative value placed on the competing alternatives. In addition, students' choice to delay gratification depends upon their expected likelihood of successful performance, given that they devote their time to this academic goal instead of a more immediate reward. Bembenutty and his colleagues (Bembenutty, 1999, 2008，2009; Bembenutty \& Karabenick, 1998) have demonstrated a relationship between academic delay of gratification and several motivational determinants. For example, Bembenutty (2008) found that college students were more likely to engage in academic delay of gratification when they liked the delay alternative, considered the delay alternative more important than the nondelay alternatives, and had higher expectations that the delay alternative would provide better outcomes than the non-delay alternatives. Further, after controlling for gender, importance of the delay alternative versus immediate alternative was a significant predictor of academic delay of gratification.

\section{Academic delay of gratification and academic achievement}

There is compelling research evidence that individual differences in children's delay preferences are associated with subsequent higher academic achievement, intelligence, and the need for achievement (Mischel, 1961; Mischel et al., 1988). Bembenutty and Karabenick (1998) reported that academic delay of gratification correlated positively with expected and obtained final course grades in a sample of college students. Bembenutty (2007) found a positive relationship between academic delay of gratification and final course grade for Caucasian male and female college students. Bembenutty (2009) demonstrated that the positive relationship between academic delay of gratification and academic achievement held even after controlling for the effects of students' ratings of the course, expected grades, and the degree of interest, importance, and utility of the academic task.

\section{Academic delay of gratification and study time allocation}

Bembenutty and his colleagues (Bembenutty, 2007, 2009; Bembenutty \& Karabenick, 1998) reported a positive association among college students between academic delay of gratification, time management and study environment. However, there is insufficient evidence of a link between academic delay of gratification and self-reported intentions and behavior that is indicative of academic delay of gratification, such as the actual time high school students devote to their study. In a recent study, Zhang, Karabenick, Maruno, and Lauermann (2011) assessed Chinese elementary school children's willingness to delay gratification, and the time they devoted to non-school study and playtime during an extended interval prior to taking a high stakes final exam. They found that children who exhibited a higher willingness to delay gratification were more likely to spend time studying and less time playing several weeks prior to the exam in contrast to those children with a lower willingness to delay gratification.

\section{Aim and rational of the present study}

Several studies have sought to investigate the relationships of academic delay of gratification to motivational determinants, academic achievement, and management of study time in college student samples using correlational and regression analyses techniques despite the fact that these statistical procedures do not provide a complete picture of any intercausal connections among the variables (Pedhazur, 1997).

Furthermore, although early writings on academic delay of gratification are rooted in cross-cultural psychology (Gallimore, Weiss, \& Finney, 1974), the vast majority of academic delay of gratification research has been conducted in Western cultures. Thus, there is a need for more research to be conducted on how participants from different cultures construe academic delay of gratification, and how academic delay of gratification is related to other psychological constructs within nonWestern cultures.

Given these insights/perceptions, the present study extends the existing literature on the relationships of academic delay of gratification to motivational determinants, academic achievement, and management of study time in several ways. Firstly, the present study explores the relationships among these variables in a non-Western, Middle Eastern Arab cultural context and as such, it provides evidence of the applicability of motivational constructs 
based on the theories largely developed in the West. The cultural variation in construing human behavior important for the study of academic delay of gratification because some cultures may be more conducive to academic delayed gratification than others. This may be true because cultures differ in educational opportunities, available attractive alternative activities that are typical features of learning contexts, and the values associated with academic tasks. For example, in societies with stronger collectivistic values, students have greater filial allegiances that result in stronger incentives for higher academic performance and academic delay of gratification (Ratner \& Hui, 2003).

Secondly, the present study provides a test of a theoretical model combining variables derived from the expectancy-value theory and the theory of self-regulated learning using a path analysis technique. One of the strengths of the path analysis is that it estimates a system of equations that specifies all the possible causal linkages among a set of variables. In addition, path analysis enables researchers to break down or deconstruct correlations among variables into causal (i.e., direct and indirect) and noncausal (e.g., superious) components. Thus, path analysis helps researchers disentangle the complex interrelationships among variables and identify the most significant pathways involved in predicting an outcome. Furthermore, researchers using nonexperimental, quantitative, or correlational data can test whether their hypotheses regarding the relationships among variables are plausible and supported by the data and represent underlying (causal) processes (Pedhazur, 1997).

Thirdly, although the findings of several studies have shown that college students higher in academic delay of gratification are more likely to manage their time and study environment, there is insufficient evidence for the link between academic delay of gratification and selfreported intentions and behavior that is indicative of academic delay of gratification, such as the actual time students devote to their study. One of the most important issues, especially for high school students, would be whether students higher in academic delay of gratification devote adequate out-of-school time to academic tasks. This may be true given that high school represents a highly competitive and academically demanding educational stage that contributes decisively towards students' academic and career future.

To summarize, the aim of the present study is to test a path analysis model that can articulate the relationship of academic delay of gratification to motivational determinants, academic achievement, and study time allocation in a sample of Omani high school students. It was predicted that: (a) motivational determinants will be positively related to academic delay of gratification, (b) academic delay of gratification will be positively related to academic achievement, (c) academic delay of gratification will be positively related to study time allocation, (d) academic delay of gratification will mediate the relationship between motivational determinants and academic achievement, and (e) academic delay of gratification will mediate the relationship between motivational determinants and study time allocation. Given that motivational determinants are expected to predict academic delay of gratification, and academic delay of gratification, in turn, is expected to predict academic achievement and study time allocation, it is possible that academic delay of gratification mediates the relationship between the antecedents and the consequences.

\section{Participants}

A total of 200 Omani students (110 males and 90 females) from 5 public secondary schools in four governorates in Oman (Musandam, Muscat, Ad Dakhiliyah, and Dhofar) participated in this study. All participant students were at Year 11 and were chosen using a multistage stratified sampling strategy. All schools were located in metropolitan areas and had singlegender populations (three male schools and two female schools). The mean sample ages were $16.68(\mathrm{SD}=.76)$ and $16.23(\mathrm{SD}=.44)$ for boys and girls, respectively. Only students with complete data were retained for the present study. The percentage of missing data was $2 \%$ which represents those students who left several items blank on the Academic Delay of Gratification Scale (ADGS) and the Motivational Determinants Scale (MDS). The analysis of demographic data showed that participant students were from the same ethnic background and that $97 \%$ of them were from the working and lower social class strata. Arabic was the native language of all participant students. 


\section{Measurements}

\section{Academic delay of gratification}

Bembenutty and Karabenick (1998) developed the 10-item ADGS to assess college students' tendencies to delay gratification within specific academic situations. For each situation, the students rated their preference for an option that offered immediate gratification, such as "Miss several classes to accept an invitation for a very interesting trip" or a delay gratification option such as "Delay going on the trip until the course is over." Students responded to each item on a 4-point scale: Definitely choose $A$; Probably choose A; Probably choose B, or Definitely Choose B. Abd-El-Fattah and Al-Nabhani (2012) translated the ADGS from English into Arabic using a sample of 195 Year 11 students in Oman. They reported that an exploratory factor analysis with principal components of responses retained a 10-item single factor $($ Cronbach's alpha $=.87)$

\section{Motivational determinants}

Students were asked to report how strongly they agreed or disagreed with statements that described motivation-related features of the delayed and immediately-available alternatives. These motivational-related features were as follows: Liking (e.g., "This is something that I would like to do"); Importance (e.g., "This is something that is important to $\mathrm{me}^{\prime \prime}$ ); Expectancy (e.g., "This is something that would help me to achieve my academic goals"); Utility (e.g., "This is something that can be useful to me"), Negative Consequences (e.g., "This is something that can have negative consequences to $\mathrm{me}^{\prime \prime}$ ), and Time/Effort (e.g., "This is something that can be costly in time or effort to me). Students responded to all items of the Motivational Determinants Scale on a 4-point Likert-type scale ranging from 1 (Strongly Disagree) to 4 (Strongly agree). Differences in scores between motivation for the delay and immediate preferences were obtained by subtracting responses to the immediate alternative from the delay alternative for the four items (e.g., liking of the delay alternative minus liking for the immediate alternative $=$ difference in liking). Higher scores were thus indicative of greater liking, importance, expectancy, utility, and time/effort for the delay versus non-delay alternatives. Scores on these motivational features were summed up to form a single index of motivational deter- minants (Eccles, Wigfield, Harold, \& Blumenfeld, 1993).

\section{Academic achievement}

Students' academic achievement scores were obtained from their school records at the end of the academic year. These were the courses aggregated total scores, that is, the sum of oncourse assignments and midterm and final examination scores and were expressed as a percentage.

\section{Study hours}

Students were requested to respond to one question concerning their study time allocation "On average, how many hours a day do you spend studying?".

\section{Procedure}

Approval was obtained to conduct the research investigation at the schools prior to data collection. Students were recruited to participate in the present study during their normal classes at their schools. All students agreed by signing a consent form prior to their participation in the present study that stated that they were willing to respond to the ADGS, the Motivational Determinants Scale, and one question concerning their study time allocation. The consent form also indicated that participant students agreed that their end-of academic year achievement scores could be obtained from their school records. Students first responded to the ADGS, then the Motivational Determinants Scale, and finally to the question concerning study time allocation. The measures were administered by trained according to standardized instructions. Students were informed that participation was voluntary and that confidentiality of their answers would prevail at all times. Only certain classes in each school participated in the present study depending on students' classroom schedules on the day and time of the administration of the measures. Students completed the three measures in 15 to 20 minutes.

\section{Path analysis}

Given that the data appear normally distributed at univariate and multivariate levels, the full information maximum likelihood estimation was used to analyze the variancecovariance matrices and estimate the path analysis model parameters and obtain fit indexes using the path analysis technique (Byr- 
ne, 2010; Kline, 1998). The AMOS 7.0 program (Arbuckle, 2006) was used to run all analyses. In this path analysis model, depicted in Figure 1, motivational determinants were set as a positive predictor of academic delay of gratification, and academic delay of gratification was set as a positive predictor of academic achievement and study hours. Several absolute and relative goodness-of-fit indexes were used to evaluate the path model's goodnessof-fit to the data. Absolute fit indices included Chi-square $\left(X^{2}\right)$, Standardized Root MeanSquare Residual (SRMR), and Root-MeanSquare Error of Approximation (RMSEA). Relative fit indices included Comparative Fit Index (CFI) and Nonnormed Fit Index (NNFI). When modeling normally distributed data, SRMR values of approximately .08 or below, RMSEA values of approximately .06 or below, CFI values of approximately .95 or above, and NNFI of approximately .95 or higher suggest adequate model-data fit (Byrne, 2010; Hu \& Bentler, 1998). Because the $X^{2}$ is sensitive to sample size, Hoelter (1983) recommended reporting the $\chi^{2} / \mathrm{df}$ ratio and suggested that ratios below 2.0 indicate a reasonable fit.

The analysis showed that the path model fitted the data adequately $\left(\chi^{2}=5.31, \mathrm{df}=3 ; \chi^{2} / \mathrm{df}\right.$ $=1.77$, RMSEA = .04 (CI. $.02-.07)$, CFI = .98, SRMR $=.06, \mathrm{NNFI}=.97)$. The full set of significant paths is presented in Figure 2 along with the associated variance explained $\left(R^{2}\right)$ for each criterion variable. In line with our hypotheses, the analysis showed that motivational determinants positively predicted academic delay of gratification $(\beta=.37)$. Academic delay of gratification positively predicted academic achievement $(\beta=.32)$ and study hours $(\beta=$ .28 ,). Motivational determinants positively predicted academic achievement $(\beta=.29)$ and study hours $(\beta=.33)$.

Table 1

Descriptive statistics, Pearson's correlation, and Cronbach's alpha for motivational determinants, academic delay of gratification, academic achievement, and study hours

\begin{tabular}{|c|c|c|c|c|c|c|c|c|c|}
\hline Variable & 1 & 2 & 3 & 4 & $\mathrm{M}$ & SD & $\begin{array}{l}\text { Skew- } \\
\text { ness }\end{array}$ & $\begin{array}{l}\text { Kurto- } \\
\text { sis }\end{array}$ & $\begin{array}{l}\text { Cronbach's } \\
\text { alpha }\end{array}$ \\
\hline $\begin{array}{l}\text { 1. Motivational determi- } \\
\text { nants }\end{array}$ & - & & & & 3.1 & .85 & 1.33 & .95 & .84 \\
\hline $\begin{array}{l}\text { 2. Academic delay of } \\
\text { gratification }\end{array}$ & .35 & - & & & 3.3 & .73 & 1.12 & .83 & .89 \\
\hline 3. Study hours & .30 & .32 & - & & 4.4 & .80 & .63 & .22 & NA \\
\hline 4. Academic achievement & .39 & .36 & .34 & - & 86.6 & 1.7 & 1.51 & 1.20 & NA \\
\hline
\end{tabular}

Note. NA = Not applicable

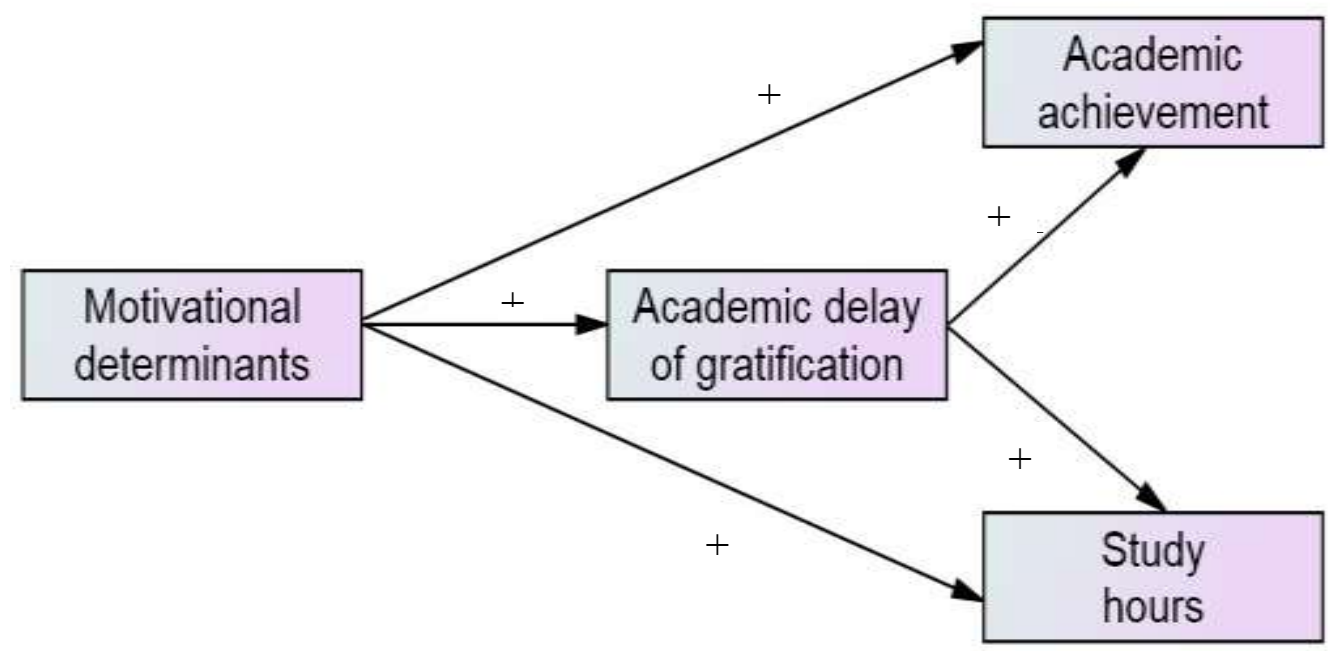

Figure 1

A hypothesized path analysis model of the relationships among motivational determinants, academic delay of gratification academic achievement, and study hours (positive ' + ' indicates a positive effect of an independent variable on a dependent variable when all other independent variables in the model are held constant) 


\section{Mediation analysis}

Given the findings of the path analysis model, we proceeded to test whether academic delay of gratification mediates the relationship between motivational determinants and academic achievement and study hours. In this analysis, we simultaneously regressed academic achievement and study hours on academic delay of gratification and on motivational determinants. Gender was set as a covariate. We ran the mediation analysis using the SPSS script that accompanies the paper by Preacher and Hayes (2008) on the usage of the bootstrapping method to test mediation models. The bootstrapping method involves repeated random repeated random sampling observations with replacement from the dataset to compute the desired statistic in each resample (Chernick, 1999). In the present study, we set the estimation convergent index to 1000 bootstrap samples to allow for the convergence of the indirect effect estimates. The bootstrapped estimates of the indirect effects, along with the bias-corrected accelerated (BCA) 95\% confidence intervals $(\mathrm{CI})$, were calculated.

The analysis showed a significant indirect effect of motivational determinants on academic achievement through academic delay of gratification (indirect effect $=.13$; BCA $95 \%$ CI lower bound $=0.10$, BCA $95 \%$ CI upper bound $=$ 0.17). This finding indicated that academic delay of gratification mediates the relationship between motivational determinants and academic achievement. The analysis also showed a significant indirect effect of motivational determinants on study hours through academic delay of gratification (indirect effect $=.11$; BCA 95\% CI lower bound =0.08, BCA 95\% CI upper bound $=0.15$ ). This finding indicates that academic delay of gratification mediates the relationship between motivational determinants and study hours.

\section{Discussion}

The aim of the present study was to investigate the relationships among motivational determinants, academic delay of gratification, academic achievement, and study hours and whether academic delay of gratification mediates the relationship between motivational determinants and academic achievement and study hours. Descriptive analyses showed that females reported higher tendencies of academic delay of gratification than males. This find- ing is in line with the findings reported by Bembenutty and his colleagues (Bembenutty, 2007, 2009; Bembenutty \& Karabenick, 1998) using samples of college students. This finding is noteworthy since it suggests that males and females can show differences in the way they manage academic situations by remaining goal oriented and committed to tasks while facing distractions that are typical features of learning contexts. However, this finding should not be interpreted as suggesting that males and females are inherently different in ways that lead them to engage in academic endeavors in a particular way. The socialization process and classroom contexts, including academic tasks, reward structures, instructional methods, and instructors' behaviors, may be associated with the patterns of the academic behavior reported by the students in the present study.

The path analysis showed that motivational determinants were positively related to academic delay of gratification. This finding firmly embeds academic delay of gratification within the framework of the expectancy-value theory (Eccles \& Wigfield, 2002; Eccles et al., 1993) and the motivational view of delay of gratification (Mischel, 1996). Within hindsight, this appears to be a readily understood relationship: students' willingness to delay gratification in order to pursue long-term academic goals is associated with their motivationrelated judgments of delay vs. non-delay alternatives as articulated by incentive value, such as the benefits, or rewards associated with the academic alternatives and the tempting alternatives. Thus, an expectancy-value mechanism seems to underlie the subjective calculation and ultimately the decision of whether the value and feasibility of attaining a delayed reward, relative to the value of the immediately available one, is high enough to warrant a choice between waiting or working to attain it. This finding is consistent with the findings of Bembenutty and his colleagues (Bembenutty, 1999, 2008, 2009; Bembenutty \& Karabenick, 1998). For example, Bembenutty (2009) reported that value based incentives were positively associated with how important, useful, and interesting college students perceived the delay alternative to be, but were inversely related to students' consideration of negative consequences associated with 
possible selection of the immediate alternatives versus delay alternatives.

The path analysis also showed that academic delay of gratification is positively related to academic achievement and study hours. This relationship can be explained within the framework of the self-regulated learning theory because academic delay of gratification has commonly been conceptualized as involving successful self-regulated learning (Bembenutty \& Karabenick, 1998; Zimmerman, 1998). According to Bembenutty $(2007,2009)$, successful self-regulated learners engage in academic delay of gratification by deferring attractive activities in order to achieve long-term goals. Those students also orchestrate their study environment to serve an adaptive purpose that facilitates academic achievement and selfimposed constraints of their own actions in order to devote more time to their study. In contrast, less-skilled self-regulated learners engage in immediate gratification that could preclude them from academic success. Mischel (1996) conceptualized the ability to delay gratification as part of the self-regulatory system necessary to guide behavior without external controlling stimuli. He suggested that the ability to delay gratification is a process of a self-regulatory system of willpower that orchestrates repetitive-used this word before maintenance of motivation and engagement in goals. Zimmerman (1998) argued that lessskilled self-regulated learners "must generate extraordinary personal motivation to delay of gratification until distal goals are achieved." (p. 6). In line with this finding, Bembenutty and his colleagues reported a positive relationship between academic delay of gratification and the control of time and study environment (Bembenutty, 2007; Bembenutty \& Karabenick, 1998) and that of academic achievement (Bembenutty, 2007, 2009; Bembenutty \& Karabenick, 1998).

The mediation analysis also showed that academic delay of gratification mediated the relationship between motivational determinants and academic achievement and study hours. Specifically, motivational determinants were positively associated with academic delay of gratification which in turn was positively associated with academic achievement and study hours.

The major limitation of the present study was the cross sectional nature of the data. As a re- sult, definitive conclusions about the relationship among motivational determinants, academic delay of gratification, academic achievement, and study hours could not be drawn. A different method for understanding the developmental precursors and consequences of academic delay of gratification would be to examine them over time rather than at a single point in time. The second limitation was the use of self-reported measures of motivational determinants, academic delay of gratification, and study hours. Although selfratings of these constructs remain the standard used by most studies, future studies should assess behaviors associated with these constructs either as observed by others or by researchers' direct observations. Until such studies are conducted, these constructs will remain largely defined as cognitive self-construal processes rather than observable traits.

To summarize, the current findings provide further insight into the dynamics which underpin students' academic delay of gratification. It could be argued that motivational determinants are associated indirectly and positively with academic achievement and study hours because they encourage positive mediating factors (i.e., academic delay of gratification) that facilitate important educational outcomes.

\section{References}

Abd-El-Fattah, S. M., \& Al-Nabhani, H. Z. (2012). From self-theories of intelligence to academic delay of gratification: The mediating role of achievement goal. Australian Journal of Educational and Developmental Psychology, 12, 93 - 107.

Arbuckle, J. L. (2006). AMOS (Version 7.0) [Computer Program]. Chicago, IL: SPSS.

Bembenutty, H. (1999). Sustaining motivation and academic goals: The role of academic delay of gratification. Learning and Individual Differences, 11, 233-257.

Bembenutty, H. (2007). Self-regulation of learning and academic delay of gratification: Gender and ethnic differences among college students. Journal of Advanced Academics, 18, 586-616.

Bembenutty, H. (2008). Academic delay of gratification and expectancy-value. Personality and Individual Differences, 44, 193202. 
Bembenutty, H. (2009). Academic delay of gratification, self-regulation of learning, gender differences, and expectancy value. Personality and Individual Differences, 46, 347-352.

Bembenutty, H., \& Karabenick, S. A. (1998). Academic Delay of Gratification. Learning and Individual Differences, 10, 329-346.

Chernick, M. R. (1999). Bootstrap methods: A practitioner's guide (Wiley series in probability and statistics). New York, NY: Wiley.

Cohen, J. (1988). Statistical power analysis for the behavioral sciences ( $2^{\text {nd }}$ ed.). Hillsdale, NJ: Lawrence Erlbaum Associates.

DeCarlo, L. (1997). On the meaning and use of kurtosis. Psychological Methods, 2, 292-307.

Eccles, J. S. (2005). Subjective task value and the Eccles et al. model of achievement related choices. In A. J. Elliot \& C. S. Dweck (Eds.), Handbook of competence and motivation (pp. 105-121). New York, NY: Guilford Press.

Eccles, J. S. (2007). Motivational perspective on school achievement: Taking responsibility for learning and teaching. In R. J. Sternberg \& R. F. Subotnik (Eds.), Optimizing student success in schools with the new three Rs (pp. 199-202). Charlotte, NC: Information Age.

Eccles, J. S., \& Wigfield, A. (2002). Motivational beliefs, values, and goals. Annual Review of Psychology, 53, 109-132.

Eccles, J. S., Wigfield, A., Harold, R., \& Blumenfeld, P. B. (1993). Age and gender differences in children's self- and task perceptions during elementary school. Child Development, 64, 830-847.

Finney, S. J., \& DiStefano, C. (2006). Nonnormal and categorical data in structural equation models. In G. R. Hancock \& R. O. Mueller (Eds.), A second course in structural equation modeling (pp. 269-314). Greenwich, CT: Information Age.

Funder, D. C., Block, J. H., \& Block, J. (1983). Delay of gratification: Some longitudinal personality correlates. Journal of Personality and Social Psychology, 44, 1198-1213.

Gallimore, R., Weiss, L. B., \& Finney, R. (1974). Cultural differences in delay of gratification: A problem of behavior classification.
Journal of Personality and Social Psychology, $30,72-80$.

Hair, J. F. Jr., Anderson, R. E., Tatham, R. L. \& Black, W. C. (1995). Multivariate Data Analysis ( $3^{\text {rd }}$ ed.). New York, NY: Macmillan.

Hoelter, J. W. (1983). The analysis of covariance structures: Goodness-of-fit indices. Sociological Methods and Research, 11, 325344.

Hu, L. T., \& Bentler, P. M. (1998). Fit indices in covariance structure modeling: Sensitivity to underparameterized model misspecification. Psychological Methods, 3, 424-453.

Kline, R. (1998). Principles and practice of structural equation modeling. New York, NY: Guilford Press.

Mischel, W. (1961). Preference for delayed reinforcement and social responsibility. Journal of Abnormal and Social Psychology, $62,1-7$.

Mischel, W. (1981). Metacognition and the rules of delay. In J. H. Flavell \& L. Ross (Eds.), Social cognitive development: Frontiers and possible futures (pp. 240-271). Cambridge, England: Cambridge University Press.

Mischel, W. (1996). From good intentions to willpower. In P. M. Gollwitzer \& J. A. Bargh (Eds.), The psychology of action: Linking cognitions and motivation to behavior (pp. 197-218). New York, NY: Guilford Press.

Mischel, W., Ebbesen, E. B., \& Zeiss, A. R. (1972). Cognitive and attentional mechanisms in delay of gratification. Journal of Personality and Social Psychology, 21, 204218.

Mischel, W., \& Metzner, R. (1962). Preference for delayed reward as a function of age, intelligence, and length of delay interval. Journal of Abnormal and Social Psychology, $64,425-431$.

Mischel, W., Shoda, Y., \& Peake, P. K. (1988). The nature of adolescent competencies predicted by preschool delay of gratification. Journal of Personality and Social Psychology, 54, 687-696.

Pedhazur, E. J. (1997). Multiple regression in behavioral research (3rd ed.). Fort Worth, TX: Harcourt Brace. 
Preacher, K. J., \& Hayes, A. F. (2008). Asymptotic and resampling strategies for assessing and comparing indirect effects in multiple mediator models

Behavior Research Methods, 40, 879-891.

Ratner, C., \& Hui, L. (2003). Theoretical and methodological problems in cross-cultural psychology. Journal for the Theory of Social Behavior, 67-94, 33.

Villarroel, J. R. (2008). An examination of the psychometric properties of the Spanish version of the academic delay of gratification scale. Advances in Applied Developmental Psychology, 2, 156-167.

Wigfield, A., \& Eccles, J. S. (2000). Expectancyvalue theory of achievement motivation. Contemporary Educational Psychology, 25, 68-81.

Zhang, L., Karabenick, S. A., Maruno, S., \& Lauermann, F. (2011). Academic delay of gratification and children's study time allocation as a function of proximity to consequential academic goals. Learning and Instruction, 21, 77-94.

Zimmerman, B. J. (1998). Developing selffulfilling cycles of academic regulation: An analysis of exemplary instructional models. In D. H. Schunk \& B. J. Zimmerman (Eds.), Self-regulated learning: From teaching to self-reflective practice (pp. 1-19). New York, NY: Guilford. 